\title{
La fiesta vecinal como práctica híbrida entre el arte, la educación, la investigación y la salud comunitaria
}

\author{
The neighborhood party as a hybrid practice between art, education, \\ community health and research
}

\author{
Silvia Siles Moriana \\ Universidad Complutense de Madrid (España) \\ ssiles@ucm.es \\ Mar Castillejo Higueras \\ Universidad Complutense de Madrid (España) \\ marcastillejohigueras@gmail.com
}

Recibido 10/09/2020 Revisado 10/11/2020

Aceptado 17/11/2020 Publicado 30/11/2020

\section{Resumen:}

En torno a la organización, programación y celebración de la fiesta vecinal como práctica artística contemporánea transformadora, se plantea la creación de un fotoensayo con las imágenes de las fiestas vecinales que han tenido lugar en el contexto de los proyectos Bulevar y ARTYS, La Experimental. Ambos se desarrollaron en los espacios públicos de los distritos madrileños de Puente de Vallecas y Villaverde entre 2017 y 2019. Tanto el proyecto Bulevar, como ARTYS, La Experimental, son propuestas de intervención comunitaria en salud a través de las prácticas artísticas como herramientas para impulsar la participación activa y mejorar la calidad de vida de los barrios de San Diego y la Colonia Experimental, respectivamente. Se enmarcan dentro del proyecto institucional Arte, salud y cuidados del Servicio de Prevención y Promoción de la Salud del Organismo Autónomo de Madrid Salud dependiente del Ayuntamiento de Madrid y se articulan mediante la metodología de investigación basada en las artes, concretamente en la a/r/tografía. Las experiencias que se incluyen en este fotoensayo son procesos híbridos entre el arte, la educación, la salud comunitaria y la investigación, que, teniendo como protagonista dos comunidades, dos contextos y dos proyectos diferentes, presentan intersecciones en la experiencia vivida que supone una fiesta vecinal en el espacio público.

\footnotetext{
Sugerencias para citar este artículo,
}

Siles Moriana, Silvia; Castillejo Higueras, Mar (2020). La fiesta vecinal como práctica híbrida entre el arte, la educación, la investigación y la salud comunitaria. Tercio Creciente (Monográfico extraordinario III), págs. 7583, https://dx.doi.org/10.17561/rtc.extra3.5732

SILES MORIANA, SILVIA; CASTILLEJO HIGUERAS, MAR (2020) La fiesta vecinal como práctica híbrida entre el arte, la educación, la investigación y la salud comunitaria. Tercio Creciente (Monográfico extraordinario III), noviembre 2020, pp. 75-83, https://dx.doi.org/10.17561/rtc.extra3.5732 


\begin{abstract}
:
Concerning the organization, scheduling and celebration of the neighborhood party as a transformative contemporary artistic practice, it is proposed the creation of a photo essay with the images of the neighborhood festivals that had taken place in the context of the Bulevar project and ARTYS, La Experimental. Both were developed from 2017 to 2019 at public spaces of district of Puente de Vallecas and Villaverde, both located in Madrid. Both the Bulevar project, and ARTYS, La Experimental, are proposals for community intervention in health through artistic practices as tools to promote active participation and to improve the quality of life in San Diego and Colonia Experimental neighborhoods, respectively. These projects are framed within the institutional project Art, Health and Care of Prevention and Health Promotion Service of the Autonomous Organization of Madrid Salud dependent on the Madrid City Council. They are articulated through the Art-Based Research metodology, specifically in $\mathrm{a} / \mathrm{r} /$ photography. The experiences that include this photo essay are hybrid processes between art, education, community health and research, that having two communities, two contexts and two different projects as main factors, present intersections in the lived experience that is a neighborhood party in public space.
\end{abstract}

Palabras Clave: Arte y salud, fiesta vecinal, salud comunitaria, investigación basada en las artes

Key words: Art and Health, neighborhood party, community health, Art-Based Research

Sugerencias para citar este artículo,

Siles Moriana, Silvia; Castillejo Higueras, Mar (2020). La fiesta vecinal como práctica híbrida entre el arte, la educación, la investigación y la salud comunitaria. Tercio Creciente (Monográfico extraordinario III), págs. 7583, https://dx.doi.org/10.17561/rtc.extra3.5732

SILES MORIANA, SILVIA; CASTILLEJO HIGUERAS, MAR (2020) La fiesta vecinal como práctica híbrida entre el arte, la educación, la investigación y la salud comunitaria. Tercio Creciente (Monográfico extraordinario III), noviembre 2020, pp. 75-83, https://dx.doi.org/10.17561/rtc.extra3.5732 


\section{Tercio Creciente}

ISSN: $2340-9096$

https://dx.doi.org/10.17561/rtc.extra3.5732
Revista de Estudios en Sociedad, Artes y Gestión Cultural
Monográfico

Extraordinario III

Noviembre 2020

Investigación
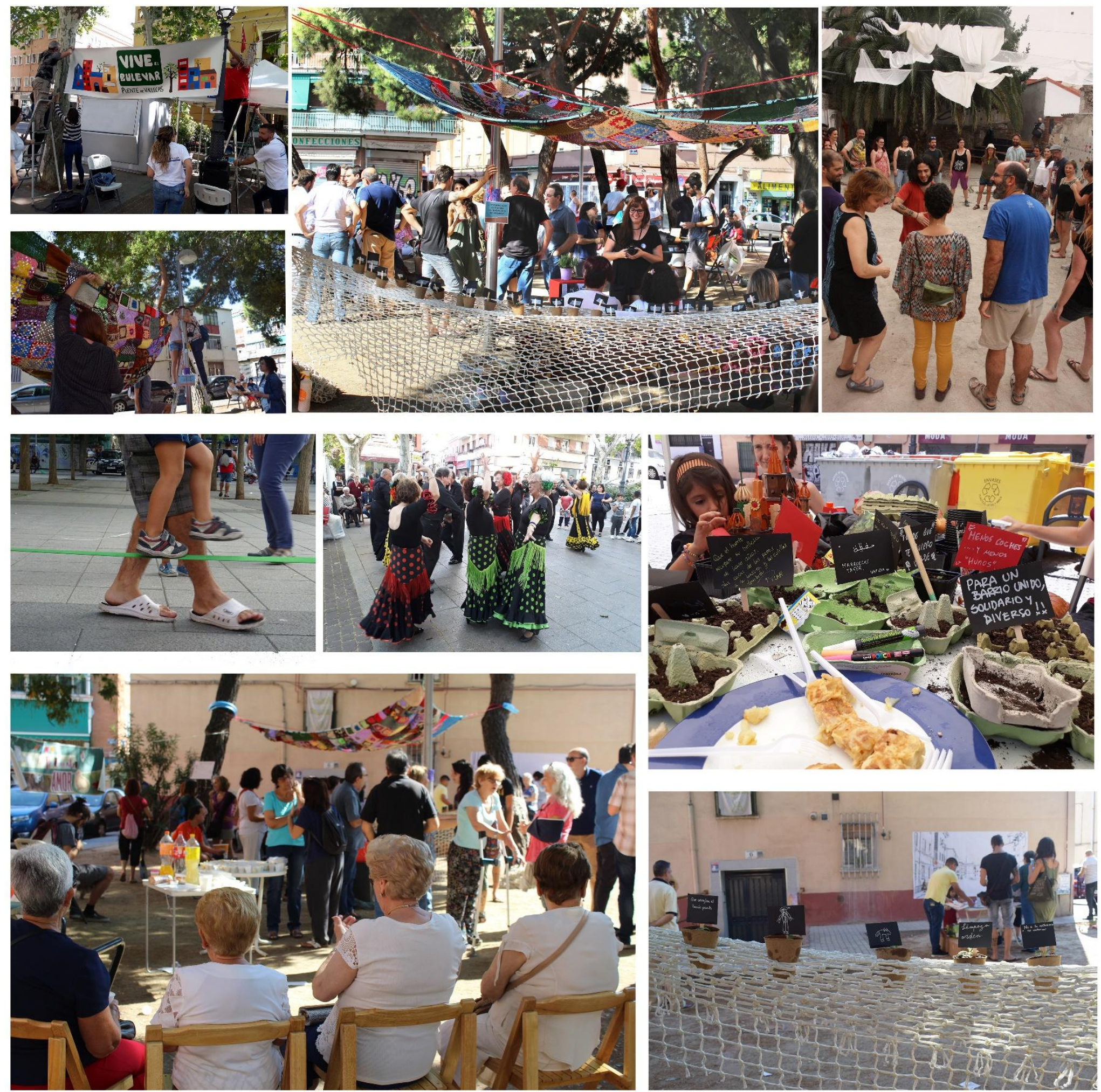

Figura 1. Fotoensayo realizado con imágenes de las fiestas vecinales del proyecto Bulevar en el barrio de San Diego de Puente de Vallecas,

y ARTYS, La Experimental, en la Colonia Experimental de Villaverde Alto. Mar Castillejo Higueras y Silvia Siles Moriana. 2017 -2019 
En torno a la organización, programación y celebración de la fiesta vecinal como práctica artística contemporánea transformadora, se plantea la creación de un fotoensayo con las imágenes de las fiestas vecinales que han tenido lugar en el contexto de los proyectos Bulevar y ARTYS, La Experimental que se desarrollaron en los espacios públicos de los distritos madrileños de Puente de Vallecas y Villaverde entre 2017 y 2019. Tanto el proyecto Bulevar, como ARTYS, La Experimental, son propuestas de intervención y participación comunitaria en salud a través de las prácticas artísticas como herramientas para impulsar la participación activa y mejorar la calidad de vida de los barrios de San Diego y la Colonia Experimental, respectivamente.

Por un lado, El Bulevar de Peña Gorbea de Puente de Vallecas es un lugar de encuentro en el Barrio de San Digo donde conviven diferentes recursos municipales, asociativos y comercios, pero, a su vez, presenta diferentes problemáticas que impiden que la comunidad pueda disfrutar del entorno. Por este motivo, el Centro Municipal de Salud Comunitaria $(\mathrm{CMSc})^{1}$, diferentes entidades y colectivos del barrio ponen en marcha una serie de eventos y actividades con el objetivo de transformar este espacio público en un lugar más saludable, a través de la creación de redes y vínculos comunitarios (Castillejo, 2019).

Por el otro, ARTYS: La Experimental es un proyecto de promoción de la salud comunitaria a través del arte como metodología para facilitar la participación de la ciudadanía y promover la mejora de la calidad de vida de la Colonia Experimental de Villaverde Alto. Se enmarca en el arte basado en la comunidad, las prácticas artísticas colaborativas y la participación ciudadana como derecho comunitario y su principal objetivo es impulsar el bienestar físico, mental y social a través de las prácticas artísticas y de una red de cuidados mutuos. Este proyecto se construye a partir de un grupo motor que se ha constituido como "comunidad creativa", formada por el Servicio de Convivencia Intercultural en Barrios, el CMSc de Villaverde y las vecinas de la Colonia. (Siles-Moriana, 2019).

\footnotetext{
${ }^{1}$ Los Centros Municipales de Salud Comunitaria (CMSc) son centros municipales especializados en la promoción de la salud y en la prevención de las enfermedades y otros problemas de salud. Por lo tanto, su objetivo no es tratar la enfermedad (algo que ya hacen los centros de atención primaria o especializada), sino que se centran en prevenirla y en ayudar a las personas, los grupos y las comunidades a conseguir estilos y condicciones de vida saludables. El Ayuntamiento de Madrid pone al servicio de los madrileños una red de dieciséis CMSc y ocho centros monográficos especializados, repartidos por la ciudad, dotados de una plantilla multidisciplinar formada por profesionales de la medicina, especialistas en ginecología, psiquiatría, pediatría, psicología o enfermería, además de trabajo social, auxiliares de salud y administración. Recuperado de: http://madridsalud.es/centros-madrid-salud-comunitaria/
} 
Ambos, se enmarcan dentro del proyecto institucional Arte, salud y cuidados $^{2}$, que nace de una histórica colaboración entre la Facultad de Bellas Artes de la Universidad Complutense de Madrid y el Servicio de Prevención y promoción de la Salud del Organismo Autónomo de Madrid Salud ${ }^{3}$ dependiente del Ayuntamiento de Madrid. Este servicio, en 2017, concede 3 becas de formación e investigación para el desarrollo de proyectos de promoción de la salud y participación comunitaria a través de las prácticas artísticas. De esta manera, los profesionales del arte colaboran junto a los profesionales sociosanitarios de los Centros Municipales de Salud Comunitaria en torno a 3 objetivos:

Utilizar la creación artística como herramienta para la prevención y la promoción de la salud; activar espacios de participación ciudadana entorno a la promoción de la salud; implicar a diferentes instituciones sociales y culturales (asociaciones, grupos, museos, escuelas, servicios sociales, etc.) en proyectos de arte y promoción de la salud. (Ávila, et al., 2019, 206)

De este modo, las experiencias que se incluyen este fotoensayo son procesos híbridos entre el arte, la educación, la salud comunitaria y la investigación.

En este sentido, "el arte puede ser una actividad vinculada a la comunidad, al contexto específico y plantea la posibilidad de colaborar en la construcción de una alternativa colectiva que participe activamente en los procesos de transformación social" (Parramón, 2003, p. 3). Por tanto, el concepto de arte basado en la comunidad o arte comunitario es el primero de los pilares centrales de estos procesos híbridos, que Palacios (2009) asocia:

(...) a un tipo de prácticas que buscan una implicación con el contexto social, que persiguen, por encima de unos logros estéticos, un beneficio o mejora social y, sobre todo, que favorecen la colaboración y la participación de las comunidades implicadas en la realización de la obra. Estas prácticas implican una revisión de los conceptos modernistas de artista y de obra de arte. El artista delega parte de sus funciones tradicionales en el grupo y el concepto de obra artística se transforma por su carácter procesual y de intervención social. (p. 199)

2 Proyecto Arte y Salud. "Proyectos en acción". Recuperado de: https://www.ucm.es/arteysaludproyectos/proyectos-en-accion-doctorado-e-investigacion

${ }^{3}$ Madrid Salud es un Organismo Autónomo del Ayuntamiento de Madrid creado en 2005 para la gestión de las políticas municipales en materia de Salud Pública y Drogodependencias dentro del término municipal. Trabajamos por la calidad del agua, la seguridad de los alimentos, la mejora de la salud ambiental y la integración sostenible de los animales domésticos en la ciudad. Madrid Salud se encarga también de la Prevención de Riesgos Laborales del Ayuntamiento de Madrid y de sus organismos autónomos. Nuestro objetivo es hacer de Madrid una ciudad cada vez más saludable promoviendo la salud en un sentido amplio y poniendo el acento en la prevención de enfermedades de la ciudadanía a través de la atención integral a las personas con adicciones y la promoción de hábitos saludables. Recuperado de: http://madridsalud.es/quienes-somos/ 
Por todo ello, la dimensión pedagógica es intrínseca a este tipo de práxis artística comunitaria. Según el artista y pedagogo Joseph Beuys, que proclamaba la creatividad como ciencia de la libertad, el proceso educativo se puede dar en cualquier lugar y escenario en los cuales se establecen diálogos articulados mediante dicha creatividad y que nos invitan a pensar en colectividad; esa creatividad involucra el pensamiento y la acción para dar sentido al mundo por lo que el arte se amplía radicalmente hacia todas las esferas de la vida social y de la actividad transformadora de los seres humanos (Gutiérrez-Galindo, 2011, p. 9).

Por otro lado, se enuncia la salud comunitaria como otro de los ejes vertebradores de las fiestas vecinales de ARTYS y el Bulevar. Se trata de un paradigma que, según López Ruíz y Padilla (2017), tiene un enfoque colectivista y emancipador donde se eliminan todos los factores individuales para la construcción de una salud desde lo colectivo. Por lo tanto, se considera a la comunidad como un foco generador de salud, ya que, no se entiende a los actores sociales como sujetos pasivos, sino que, juegan un papel fundamental en la construcción de salud colectiva como agentes de cambio. En resumen, la salud comunitaria es un modelo encaminado a empoderar a la población de un territorio, y desde esta perspectiva se considera el barrio como el ámbito de análisis e intervención para mejorar la salud (Castillejo, et al., 2018).

Por último, este trabajo se enmarca en la metodología de Investigación Basada en las Artes (IBA) o Art-Based Research (ABR), de la cual, Barone y Eisner, hacen una declaración fundamental, en su primer libro dedicado únicamente al tema:

La investigación basada en artes era -y es- un intento de utilizar las formas de pensamiento y las formas de representación que proporcionan las artes como medio a través de las cuales el mundo puede ser comprendido mejor... (Barone y Eisner, 2012, p. 11, como se citó en MarínViadel y Roldán, 2019, p. 885).

Este modo de investigar, surge a partir de lo que Piccini (2012) denomina giro narrativo en la investigación en ciencias sociales, proponiendo:

el uso de métodos y procesos creativos y artísticos para acercarse al conocimiento, donde el investigador no es meramente un observador sino también un hacedor y donde sus propias vivencias, creatividad y mirada personal, pudieran aportar nuevos insights y asistir a la creación de conocimientos, así como crear espacios nuevos de investigación. (p. 3) 
De este modo, un trabajo de investigación puede ser creativo y emotivo, y el conocimiento se puede construir y presentar a través de diferentes lenguajes, donde el objetivo "no es tanto explicar los acontecimientos educativos o sociales como proponer o sugerir nuevas maneras de verlos o comprenderlos" (Marín-Viadel \& Roldán, 2019, 885)

Por consiguiente, tanto los proyectos que se muestran en este fotoensayo, como el fotoensayo en sí mismo, se enmarcan en la a/r/tografía, un modo de hacer de la investigación basada en las artes que se ajusta a las necesidades de este tipo de proyectos (Siles-Moriana, et. al., 2020) ya que articula a un mismo nivel la dimensión artística, educativa e investigadora en prácticas colaborativas, participativas y socio-comunitarias. (Marín-Viadel y Roldán, 2019).

De este modo, se ha considerado pertinente este formato creativo para presentar la propuesta que, aun teniendo como protagonista dos comunidades, dos contextos y dos proyectos diferentes, presentan intersecciones en la experiencia vivida que supone una fiesta vecinal en el espacio público en el sentido en el que Fran Quiroga (2019) habla de ellas en su texto La fiesta, lo raro y el espacio público:

Pensar la fiesta como ese ejercicio del derecho a hacer ciudad es un compromiso más con la capacidad que tenemos de construir relaciones afectivas entre nosotrxs y también con el lugar que pisamos. Al hacer la fiesta, hacemos ciudad. Juntarse con las amistades a escuchar música o bailar un chotis es otra manera de ocuparlo, de estar juntxs. Hacer comunidad, aunque sea difusa o efímera, nos reconcilia con saber que no vivimos en soledad. Al tramar estos lugares de encuentro y deseo generamos complicidades unxs con otrxs. (p.11) 


\section{Referencias.}

Ávila, N., Azcona, C., Claver, M. D., Larraín, A., Segura, J., \& Martínez, M. (2019). Art, health promotion and community health: Constructing the 'Madrid Salud' model. Journal of Applied Arts \& Health. 10 (2), 203-217 https://doi.org/10.1386/jaah.10.2.203 1

Castillejo, M. (2019). Replanteando el Bulevar: Un jardín vertical como intervención artística y comunitaria. EARI, Educación Artística Revista de Investigación, (10), 82-94. Doi: https://doi.org/10.7203/eari.10.14246

Castillejo, M., Fernández-Cedena, J., Siles, S., Ávila, N. y Claver, M. D. (2018). Batas Nómadas en Madrid Salud: el arte y los artistas en equipos profesionales de salud comunitaria. Gaceta Sanitaria, 32(5), p. 466-472. Doi: 10.1016/j.gaceta.2018.03.008

Gutiérrez-Galindo, B. (2011). El artista educador. Los casos de Allan Kaprow y Joseph Beuys. Facultad de Arte y diseño. Universidad Autónoma de México. Recuperado de: https://www.yumpu.com/es/document/read/56702685/elartistaeducador

López Ruíz, V. y Padilla, J., (coords.). (2017). Salubrismo o barbarie. Córdoba, España: Atrapasueños editorial.

Marín-Viadel, R, y Roldán, J. (2019). A/r/tografía e Investigación Educativa Basada en Artes Visuales en el panorama de las metodologías de investigación en Educación Artística. Arte, Individuo y Sociedad, 31(4), pp. 881-895. Doi: https://dx.doi.org/10.5209/aris.63409

Quiroga, F., (coord.). (2019). La fiesta, lo raro y el espacio público. Madrid: Matadero Intermediae

Palacios, A. (2009). El arte comunitario: origen y evolución de las prácticas artísticas colaborativas. Arteterapia: Papeles de arteterapia y educación artística para la inclusión social, 4, 197-211. Recuperado de: https://revistas.ucm.es/index.php/ARTE/article/view/9641

Parramón, R. (Octubre, 2003). Arte, participación y espacio público. Models de participació en xarxa. Jornada d'innovació estratégica. Recuperado de: https://www.insumisos.com/lecturasinsumisas/Espacio\%20publico\%20y\%20participaci on.pdf 
Piccini, R. (2012). Investigación Basada en las Artes. Marco teórico para T. E. Instituto Escuela Nacional de Bellas Artes. Universidad de la República Oriental de Uruguay. Recuperado de: https://www.researchgate.net/publication/235634127_Investigacion_Basada_en_las_Art $\underline{\text { es }}$

Siles-Moriana, S. (2019). El arte y la creatividad como nuevas formas de bienestar. Primera fase de ARTYS, La Experimental, proyecto de Arte y Salud Comunitaria en la Colonia Experimental de Villaverde Alto (Madrid). EARI, Educación Artística Revista de Investigación, (10), 150-167. Doi: https://doi.org/10.7203/eari.10.12608v

Siles-Moriana, Castillejo Higueras, M., Larraín Vergara, M., Azcona Gómez, C. y FernándezCedena, J. (2020). Arte y Salud: Un espacio de encuentro creativo desde lo comunitario. Investigación transdisciplinar colaborativa, nuevas metodologías y herramientas de investigación creativa. En R. López Cano (Presidencia), INDISCIPLINES, Congrés sobre la Recerca en la Pràctica de les Arts. Comunicación llevada a cabo en el Congreso sobre la Investigación en la Práctica de las Artes, 28-30 de noviembre de 2019, Barcelona. Recuperado de: https://b3a5d73f-f80a-4b71-811cb550bd9d8e10.filesusr.com/ugd/cb4a0a_a0375d4af9de462baa4fa4d769b05529.pdf 
